\title{
ON THE ERGODICITY OF A CLASS OF REAL SKEW PRODUCT EXTENSIONS OF APPROXIMATIONS
}

\author{
G. R. GOODSON
}

\begin{abstract}
In this paper conditions are given for real skew product extensions of cyclic $K S$ approximations to be ergodic. These results are then applied to show that if $T_{\alpha}$ is an irrational rotation on the unit circle, there exists an uncountable dense collection of measurable sets for which the corresponding skew product extension is ergodic.
\end{abstract}

Introduction. Suppose that $T$ is an automorphism of the Lebesgue space $(X, \mathscr{F}, \mu)$. Let $A \in \mathscr{F}$ with $\mu(A)=\beta$, then the transformation $T_{A}: X \times \mathbf{R} \rightarrow X \times \mathbf{R}$ defined by

$$
T_{A}(x, t)=\left(T x, t+\chi_{A}-\beta\right)
$$

is called a real skew product extension of $T$, and is an automorphism of $(X \times \mathbf{R}, \mathcal{F} \times$ $\Re, \mu \times \lambda)$ where $\Re$ denotes the Lebesgue measurable sets and $\lambda$ is Lebesgue measure on $\mathbf{R}$.

Let $\alpha \in[0,1)$ be irrational and denote by $T_{\alpha}$ the irrational rotation

$$
T_{\alpha} x=x+\alpha(\bmod 1), x \in[0,1) .
$$

In [1] Atkinson and Riley showed that if $A=[0, \beta]$, then $\left(T_{\alpha}\right)_{A}$ is ergodic for almost all $\beta \in[0,1)$. The purpose of this paper is to prove the following theorem.

THEOREM 1. For fixed irrational $\alpha$, there is an uncountable dense collection of measurable sets $A$ for which $\left(T_{\alpha}\right)_{A}$ is ergodic.

In order to prove Theorem 1 we first prove a general result concerning the ergodicity of $T_{A}$ when $T$ admits a cyclic approximation (in the sense of Katok and Stepin [3]) with some speed $\theta / n$.

This work is closely related to the work of Atkinson and Riley[1] and is dependent on the results of Schmidt [4] and the ideas of Katok and Stepin [3]. The author would like to thank Dr. D. Newton for some helpful discussions and the University of Sussex, where this research was carried out, and also the Council for Scientific and Industrial Research for partial support.

1. Preliminaries. Throughout $(X, \mathscr{F}, \mu)$ is a measure space isomorphic to the unit interval with Lebesgue measure. If $A \in \mathcal{F}$, we write $A \leqslant \xi$ if $A$ is the union of members of a partition $\xi$ in $X$. 
A sequence $\{\xi(n)\}$ of partitions of $X$ converges to the unit partition, written $\xi(n) \rightarrow \xi$, provided that $\mu(A \triangle A(\xi(n))) \rightarrow 0$ as $n \rightarrow \infty$ for each $A \in \mathscr{F}$, where $A(\xi(n)) \leqslant \xi(n)$ is chosen so that $\mu(A \triangle A(\xi(n)))$ is a minimum. If $\xi(n) \rightarrow \varepsilon$ as $n \rightarrow \infty$, the sequence of partitions $\{\xi(n)\}$ is dense in $\mathscr{F}$. (Recall that $\mathscr{F}$ is a complete metric space with respect to the metric $d(A, B)=\mu(A \triangle B)$, if we identify sets which only differ on a set of measure zero.)

Definition 1. The automorphism $T: X \rightarrow X$ admits a cyclic $K S$ approximation with speed $h(n)$ if there exists a sequence of partitions $\{\xi(n)\}$ with $\xi(n)=\left\{C_{i}(n)\right.$ : $1 \leqslant i \leqslant q(n)\}$ such that

(i) $\xi(n) \rightarrow \varepsilon$ as $n \rightarrow \infty$;

(ii) $\mu\left(C_{i}(n)\right)=1 / q(n), 1 \leqslant i \leqslant q(n)$;

(iii) $\sum_{i=1}^{q(n)} \mu\left(T C_{i}(n) \Delta C_{i+1}(n)\right) \leqslant h(q(n))$ where $C_{q(n)+1}(n)$ means $C_{1}(n)$.

The above definition is from Katok and Stepin [3], as is the following definition, which enables us to approximate measurable sets by sequences of partitions.

Definition 2. A set $A \in \mathcal{F}$ can be approximated with speed $g(n)$ by a sequence of partitions $\{\xi(n)\}, \xi(n)=\left\{C_{i}(n): i=1, \ldots, q(n)\right\}$, where $\xi(n) \rightarrow \varepsilon$, if there exist sets $A(n) \leqslant \xi(n)$ such that $\mu(A \triangle A(n)) \leqslant g(q(n))$.

We briefly mention some general results concerning skew product extensions, which are to be found in Schmidt [4]. Let $T$ be an automorphism of $(X, \mathcal{F}, \mu)$ and $G$ a locally compact, second countable abelian group with Haar measure $\lambda$ and a translation invariant metric $d$.

Definition 3. Let $f: X \rightarrow G$ be measurable. The skew product $G$-extension of $T$ defined by $f$ is the transformation $T_{f}: X \times G \rightarrow X \times G$ with $T_{f}(x, y)=(T x, y+$ $f(x))$.

Definition 4. Let $f: X \rightarrow G$ be measurable. An element $y$ of $G$ is said to be an essential value of $f$ if $y=0$ or if for every $\varepsilon>0$ and every $B \in \mathcal{F}$ with $\mu(B)>0$ there exists a positive integer $k$ such that

$$
\mu\left(B \cap T^{-k} B \cap\left\{x: d\left(\sum_{j=0}^{k-1} f\left(T^{j} x\right), y\right)<\varepsilon\right\}\right)>0 .
$$

We denote the set of essential values of $f$ by $E(f)$.

The importance of $E(f)$ is indicated by the following result of Schmidt [4].

Proposition 1. Let $f: X \rightarrow G$ be a measurable function. Then

(a) $E(f)$ is a closed subgroup of $G$.

(b) $T_{f}$ is ergodic if and only if $E(f)=G$.

The following result of Atkinson and Riley [1] will be of importance in the proof of our main theorem.

Lemma 1. Let $f: X \rightarrow G$ be measurable and let $y \in G$. Supose that there is a dense squence of partitions $\xi(n), n=1,2, \ldots$, such that for every $\varepsilon>0$, there exists $a$ constant $\theta_{\varepsilon}>0$ with the property that for all sufficiently large $n$, every element $C$ of $\xi(n)$ satisfies the condition: 
There exists a positive integer $k$ with

$$
\mu\left(C \cap T^{-k} C \cap\left\{x: d\left(\sum_{j=0}^{k-1} f\left(T^{j} x\right), y\right)<\varepsilon\right\}\right) \geqslant \theta_{\varepsilon} \mu(C)
$$

then $y$ is an essential value.

2. Real skew product extensions. The following lemma is analogous to Lemma 2 of Atkinson and Riley [1]. If $y \in \mathbf{R}$ we shall use $\langle\langle y\rangle\rangle$ to denote the distance of $y$ from the nearest integer.

LEMMA 2. Suppose that $T$ admits a cyclic $K S$ approximation with respect to $\xi(n)=\left\{C_{i}(n): 1 \leqslant i \leqslant q(n)\right\}$ with speed $\theta / n$. Also suppose that $A \in \mathcal{F}$ can be approximated by $\{\xi(n)\}$ with speed $\delta / n, \delta \leqslant \frac{1}{2}$ with $\theta+2 \delta<2$.

If $\beta=\mu(A)$ and $f=\chi_{A}-\beta$ is such that $\langle\langle q(n) \beta\rangle\rangle, n=1,2,3, \ldots$, has a limit point $y \in[0, \delta)$ then $y \in E(f)$.

Proof. If $y=0, y \in E(f)$ so suppose $y \in(0, \delta)$ and that there exists a subsequence of $\langle\langle q(n) \beta\rangle\rangle$ such that $\langle\langle q(n) \beta\rangle\rangle \rightarrow y$ as $n \rightarrow \infty$, then for all $n$ large enough

$$
0<\langle\langle q(n) \beta\rangle\rangle<\delta \text {. }
$$

For each $n \in \mathrm{N}$ there is a set $A(n) \leqslant \xi(n)$ satisfying

$$
|\mu(A)-\mu(A(n))| \leqslant \mu(A \triangle A(n)) \leqslant \delta / q(n)
$$

and this implies $|q(n) \beta-| A(n)|| \leqslant \frac{1}{2}$ where $|A(n)|=\#\left\{i: C_{i}(n) \subseteq A(n)\right\}$, and hence

$$
|A(n)|-q(n) \beta= \pm\langle\langle q(n) \beta\rangle\rangle .
$$

Clearly $|A(n)|-q(n) \beta$ is either $\langle\langle q(n) \beta\rangle\rangle$ or $-\langle\langle q(n) \beta\rangle\rangle$ infinitely often. If it is the former infinitely often we will deduce that $y \in E(f)$ (if it is the latter a similar argument shows that $-y \in E(f)$, so $y \in E(f)$ since $E(f)$ is a group).

Note that the function $f_{n}=\chi_{A(n)}-\beta$ is an approximation to $f$, and for $k=$ $1,2, \ldots, q(n)$ we define sets $D_{k}(n)$ and $E_{k}(n), n=1,2, \ldots$, by

$$
D_{k}(n)=\bigcap_{j=0}^{q(n)} T^{-j} C_{k+j}(n), \quad E_{k}(n)=\bigcap_{j=0}^{q(n)-1} T^{-j}\left\{x: f(x)=f_{n}(x)\right\} \cap D_{k}(n) .
$$

It follows by standard arguments (e.g. see [3]) that

$$
\mu\left(D_{k}(n) \backslash E_{k}(n)\right) \leqslant \delta / q(n)
$$

and

$$
\mu\left(C_{k}(n) \backslash D_{k}(n)\right) \leqslant \theta / 2 q(n) .
$$

From (3) and (4) we obtain

$$
\mu\left(E_{k}(n)\right) \geqslant\{1-(\theta / 2+\delta)\} \mu\left(C_{k}(n)\right)>0 .
$$


Furthermore, if $x \in E_{k}(n)$

$$
\begin{aligned}
\sum_{j=0}^{q(n)-1} f\left(T^{j} x\right) & =\sum_{j=0}^{q(n)-1} \chi_{A(n)}\left(T^{j} x\right)-q(n) \beta \\
& =|A(n)|-q(n) \beta=\langle\langle q(n) \beta\rangle\rangle \rightarrow y \quad \text { as } n \rightarrow \infty,
\end{aligned}
$$

for some subsequence. Thus

$$
\begin{aligned}
\mu\left[C_{k}(n) \cap T^{-q(n)} C_{k}(n)\right. & \left.\cap\left\{x: \sum_{j=0}^{q(n)-1} f\left(T^{j} x\right)=\langle\langle q(n) \beta\rangle\rangle\right\}\right] \\
& \geqslant \mu\left(E_{k}(n)\right) \geqslant\{1-(\theta / 2+\delta)\} \mu\left(C_{k}(n)\right), \text { by }(5) .
\end{aligned}
$$

Since $\theta+2 \delta<1$, it follows from Lemma 1 that $y \in E(f)$.

REMARKs. Clearly by taking subsequences of $\{\xi(n)\}$ if necessary, any set $C \in \xi(n)$ may be approximated arbitrarily closely by $\xi(n+1)$-sets. More explicitly, given any $p \in \mathbf{N}$ we may assume that

$$
\mu(C \triangle C(\xi(n+1)))=o\left(\frac{1}{q(n)^{p}}\right) \text { for all } C \in \xi(n) .
$$

It follows from the above remark and the following lemma that any set $A(n) \leqslant \xi(n)$ can be approximated with arbitrary speed by $\xi(m)$-sets for $m>n$. We state the lemma without proof.

LEMMA 3. If $A(n) \leqslant \xi(n)$ and $A(n+1)$ is a best approximation to $A(n)$ by elements of $\xi(n+1)$, then

$$
\mu(A(n) \triangle A(n+1)) \leqslant \sum_{\substack{C \in \xi(n) \\ C \subseteq A(n)}} \mu(C \Delta C(\xi(n+1))) .
$$

3. Main results. Theorem 3, which is actually a corollary to Theorem 2 , is the main theorem of this section.

THEOREM 2. Let $T$ admit a cyclic $K S$ approximation with speed $\theta / n, \theta<2$. Then for almost all $\beta \in(0,1)$ there is a set $A \in \mathcal{F}$ with $\mu(A)=\beta$ such that

$$
T_{A}(x, t)=\left(T x, t+\chi_{A}-\beta\right)
$$

is ergodic.

Proof. Choose $0<\delta<\frac{1}{2}$ satisfying $\theta+2 \delta<2$, and choose a subsequence of $\{\xi(n)\}$ satisfying

$$
\mu(C \triangle C(\xi(n+1)))=o\left(\frac{1}{q(n)^{p}}\right), \text { for } C \in \xi(n)
$$

and for some fixed $p \in \mathbf{N}$ (to be specified later) and with

$$
\sum_{k=n+1}^{\infty} \frac{1}{q(k)} \leqslant \frac{1}{q(n)} \text { for } n=1,2, \ldots
$$


Let $\langle x\rangle$ denote the fractional part of $x \in \mathbf{R}$. It follows from a theorem of Hardy and Littlewood [2] (see also Weyl [5]) that the sequence $\{\langle q(n) \beta\rangle\}$ is dense in [0,1) for almost every $\beta \in[0,1)$. Choose such a $\beta$, then by taking a subsequence if necessary we may assume that $\langle q(n) \beta\rangle$ is dense in $(0, \delta / 2)$ and $0<\langle q(n) \beta\rangle<\delta / 2$.

There exist unique integers $k_{n} \in \mathbf{N}, n=1,2, \ldots$, with

$$
0<q(n) \beta-k_{n}<\delta / 2 \text { or } 0<\beta-k_{n} / q(n)<\delta / 2 q(n) \text {. }
$$

Inductively we define sets $A(n) \leqslant \xi(n), n=1,2, \ldots$, by taking $A(1)$ to be any set with $k_{1}$ elements of $\xi(1)$ (if $k_{1}=0, A(1)=\varnothing$ ). Having defined $A(n)$, take $A(n+1)$ as the union of $k_{n+1}$ elements of $\xi(n+1)$ with the property that $\mu(A(n) \triangle A(n+1))$ is a minimum. We shall show that the sequence $\{A(n)\}, n=1,2, \ldots$, constitutes a Cauchy sequence in the measure algebra $(\mathscr{F}, \mu)$.

Choose $A^{\prime}(n+1)$ to be a best approximation to $A(n)$ by $\xi(n+1)$-sets and suppose that $A^{\prime}(n+1)$ is the union of $l_{n+1}$ elements of $\xi(n+1)$. Then by Lemma 3

$$
\mu\left(A(n) \triangle A^{\prime}(n+1)\right) \leqslant o\left(\frac{1}{q(n)^{p-1}}\right)
$$

hence

$$
\left|\frac{k_{n}}{q(n)}-\frac{l_{n+1}}{q(n+1)}\right|=\left|\mu(A(n))-\mu\left(A^{\prime}(n+1)\right)\right| \leqslant o\left(\frac{1}{q(n)^{p-1}}\right) .
$$

From (2) we deduce

$$
\left|\frac{k_{n+1}}{q(n+1)}-\frac{k_{n}}{q(n)}\right|<\frac{\delta}{2 q(n)}
$$

and (3) and (4) imply that

$$
\left|\frac{l_{n+1}}{q(n+1)}-\frac{k_{n+1}}{q(n+1)}\right| \leqslant o\left(\frac{1}{q(n)^{p-1}}\right)+\frac{\delta}{2 q(n)} .
$$

It follows that we can choose $A(n+1) \leqslant \xi(n+1)$ with $k_{n+1}$ elements of $\xi(n+1)$ satisfying

$$
\begin{aligned}
\mu(A(n) \triangle A(n+1)) & \leqslant \mu\left(A(n) \triangle A^{\prime}(n+1)\right)+\mu\left(A^{\prime}(n+1) \triangle A(n+1)\right) \\
& <o\left(\frac{1}{q(n)^{p-1}}\right)+\frac{\delta}{2 q(n)},
\end{aligned}
$$

and this implies that

$$
\mu(A(n) \Delta A(m))<\sum_{k=n}^{m}\left\{o\left(\frac{1}{q(k)^{p-1}}\right)+\frac{\delta}{2 q(k)}\right\},
$$

and the convergence of the series involved (for $p$ large enough) implies that $\{A(n)\}$ is a Cauchy sequence in the complete space $\mathscr{F}$, and so there exists $A \in \mathcal{F}$ for which 


$$
\begin{aligned}
& \mu(A(n) \triangle A) \rightarrow 0 \text { as } n \rightarrow \infty \text {. Letting } m \rightarrow \infty \text { in (6) gives } \\
& \mu(A(n) \Delta A) \leqslant \sum_{k=n}^{\infty} o\left(\frac{1}{q(k)^{p-1}}\right)+\frac{\delta}{2} \sum_{k=n}^{\infty} \frac{1}{q(k)} \\
& =\sum_{k=n}^{\infty} o\left(\frac{1}{q(k)^{p-1}}\right)+\frac{\delta}{2 q(n)}+\frac{\delta}{2} \sum_{k=n+1}^{\infty} \frac{1}{q(k)} \\
& \leqslant \frac{\delta}{q(n)} \text { by }(1)
\end{aligned}
$$

for $p$ chosen suitably large.

Clearly $\mu(A)=\beta$ and $A$ can be approximated by the sequence $\{\xi(n)\}$ with speed $\delta / n$ where $\theta+2 \delta<2$. It follows from Proposition 2 that the limit points of $\langle q(n) \beta\rangle$ are in $E(f)$, i.e. $(0, \delta / 2) \subseteq E(f)$, and since $E(f)$ is a group, $E(f)=\mathbf{R}$ and so $T_{A}$ is ergodic.

THEOREM 3. Let $T$ admit a cyclic $K S$ approximation with speed $\theta / n, \theta<2$. There is an uncountable dense collection of sets $A$ in $\mathscr{F}$ for which $T_{A}$ is ergodic.

PRoof. The argument of Theorem 2 applies to almost every $\beta \in(0,1)$. If $D \in \mathscr{F}$ we can construct a set $A \in \mathscr{F}$ for which $T_{A}$ is ergodic, arbitrarily close to $D$ by firstly choosing a suitable $\beta$ close to $\mu(D)$ and then constructing the sets $A(n)$ as good approximations to $D(\xi(n))$, and then using an approximation argument.

4. Proof of Theorem 1. We now apply our result to the irrational rotation $T_{\alpha} x=x+\alpha(\bmod 1)$. Recall $(\operatorname{see}[3])$ that if $\alpha \in[0,1)$ is irrational, from the theory of continued fractions there exist irreducible fractions $p_{n} / q_{n}, n=1,2, \ldots$, such that $q_{n} \rightarrow \infty$ and $\left|\alpha-p_{n} / q_{n}\right|<1 / \sqrt{5} q_{n}^{2}$ for all $n$. This implies that $T_{\alpha}$ admits a cyclic $K S$ approximation with speed $2 / \sqrt{5} n$ with respect to the sequence of partitions $\xi(n)=\left\{C_{i}(n): 1 \leqslant i \leqslant q(n)\right\}$, where $C_{i}(n)=T_{p_{n} / q_{n}}^{i-1}\left[0,1 / q_{n}\right)$ for all $i$ and $n$. It is now clear that Theorem 1 follows since $T_{\alpha}$ satisfies the conditions of Theorem 3.

\section{REFERENCES}

1. G. Atkinson and G. W. Riley, On the ergodicity of some real line extensions of irrational rotations, Preprint, Univ. of Warwick, 1978.

2. G. H. Hardy and J. E. Littlewood, Some problems of Diophantine approximation, Acta Math. 37 (1914), 155-191.

3. A. B. Katok and A. M. Stepin, Approximations in ergodic theory, Russian Math. Surveys 22 (1967), 77-102.

4. K. Schmidt, Lectures on cocycles of ergodic transformation groups, Preprint, Univ. of Warwick, 1976.

5. H. Weyl, Über die Gleichverteilung von Zahlen mod Eins, Math. Ann. 77 (1916), 313-352.

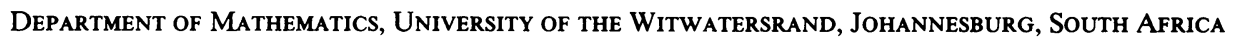

Current address: Department of Mathematics, University of Cape Town, Rondebosch, Cape 7700, South Africa 